\title{
Elucidating the Sodiation Mechanism in Hard Carbon by Operando Raman Spectroscopy
}

Julia S. Weaving ${ }^{1}$, Alvin Lim ${ }^{1}$, Jason Millichamp ${ }^{1}$, Tobias P. Neville ${ }^{1}$, Daniela Ledwoch ${ }^{1}$, Emma

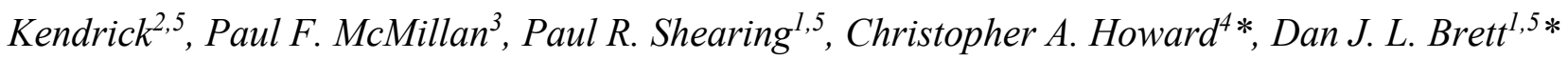
${ }^{1}$ Electrochemical Innovation Laboratory, Department of Chemical Engineering, University College London, London, WC1E 7JE, UK

${ }^{2}$ School of Metallurgy and Materials, University of Birmingham, Birmingham, B15 2TT, UK ${ }^{3}$ Department of Chemistry, University College London, London, WC1H 0AJ, UK

${ }^{4}$ Department of Physics \& Astronomy, University College London, London WC1E 6BT, UK

${ }^{5}$ The Faraday Institution, Quad One, Harwell Science and Innovation Campus, Didcot, OX11 0RA, UK AUTHOR INFORMATION

\section{Corresponding Authors}

*c.howard@ucl.ac.uk,*d.brett@ucl.ac.uk. 

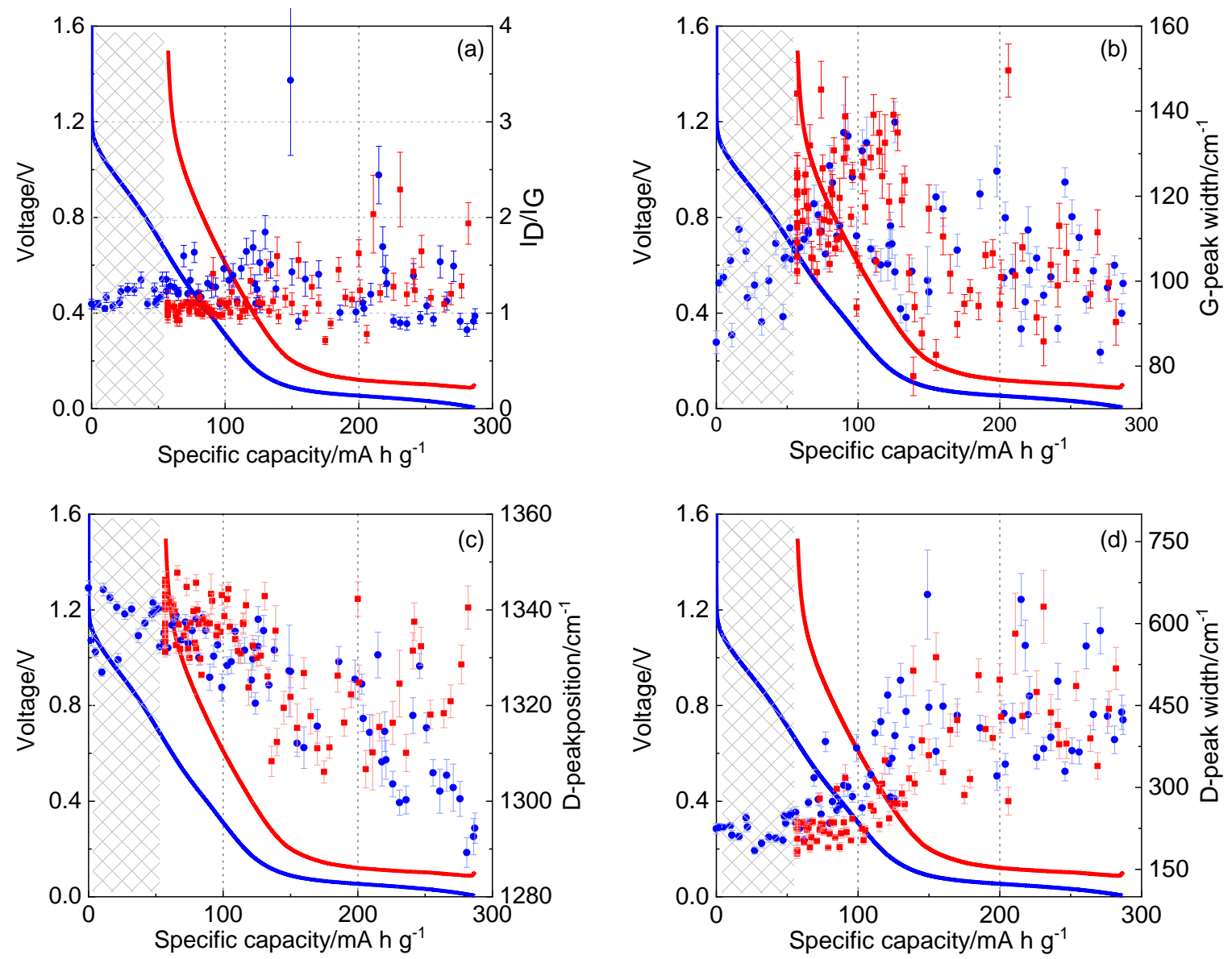

Figure S1 Operando Raman experiment for discharge (sodiation) and subsequent charge (desodiation) on cycle 1 of sodium-hard carbon cell described in Figure 3; sodiation shown in blue; desodiation shown in red; solid line $=$ voltage profile, discrete points $=$ Raman parameters from fitted G- and Dpeaks (blue dots $=$ sodiation, red squares $=$ desodiation): (a) ratio of intensities $\mathrm{I}_{\mathrm{D}} / \mathrm{I}_{\mathrm{G}}$; (b) G-peak width, (c) D-peak position, (d) D-peak width; First Cycle Loss associated with SEI formation shown in gray shading; sodiation was performed by discharging at a constant current of $\mathrm{C} / 5$ to $5 \mathrm{mV}$ (there was no constant voltage hold) and desodiation was performed by a constant current charge at $\mathrm{C} / 5$ to $1.5 \mathrm{~V} ; \mathrm{C} / 5$ was based on $300 \mathrm{~mA} \mathrm{~h} \mathrm{~g}^{-1}$ for hard carbon; G-peak position given in Figure 3. 

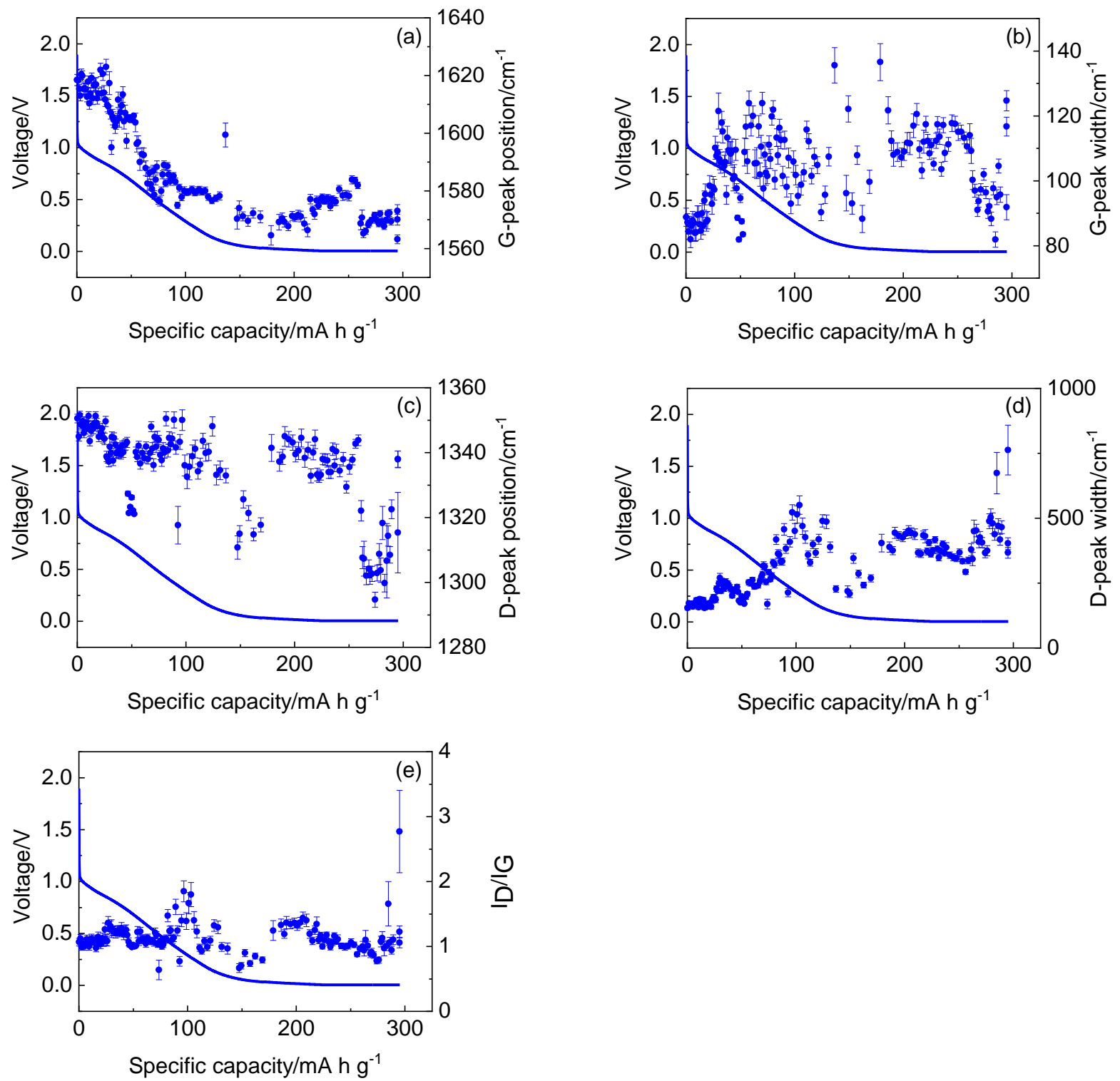

Figure S2 Operando Raman experiment for discharge (sodiation) of sodium-hard carbon cell on cycle 1; solid line $=$ voltage profile, discrete points $=$ Raman parameters from fitted G- and D-peaks: (a) Gpeak position; (b) G-peak width; (c) D-peak position; (d) D-peak width; (e) ratio of intensities $\mathrm{I}_{\mathrm{D}} / \mathrm{I}_{\mathrm{G}}$; sodiation was performed by discharging at a constant current of $\sim \mathrm{C} / 3$ to $5 \mathrm{mV}$, followed by a constant voltage hold at $5 \mathrm{mV}$ for 60 minutes; $\mathrm{C} / 3$ was based on $300 \mathrm{~mA} \mathrm{~h} \mathrm{~g}^{-1}$ for hard carbon. 

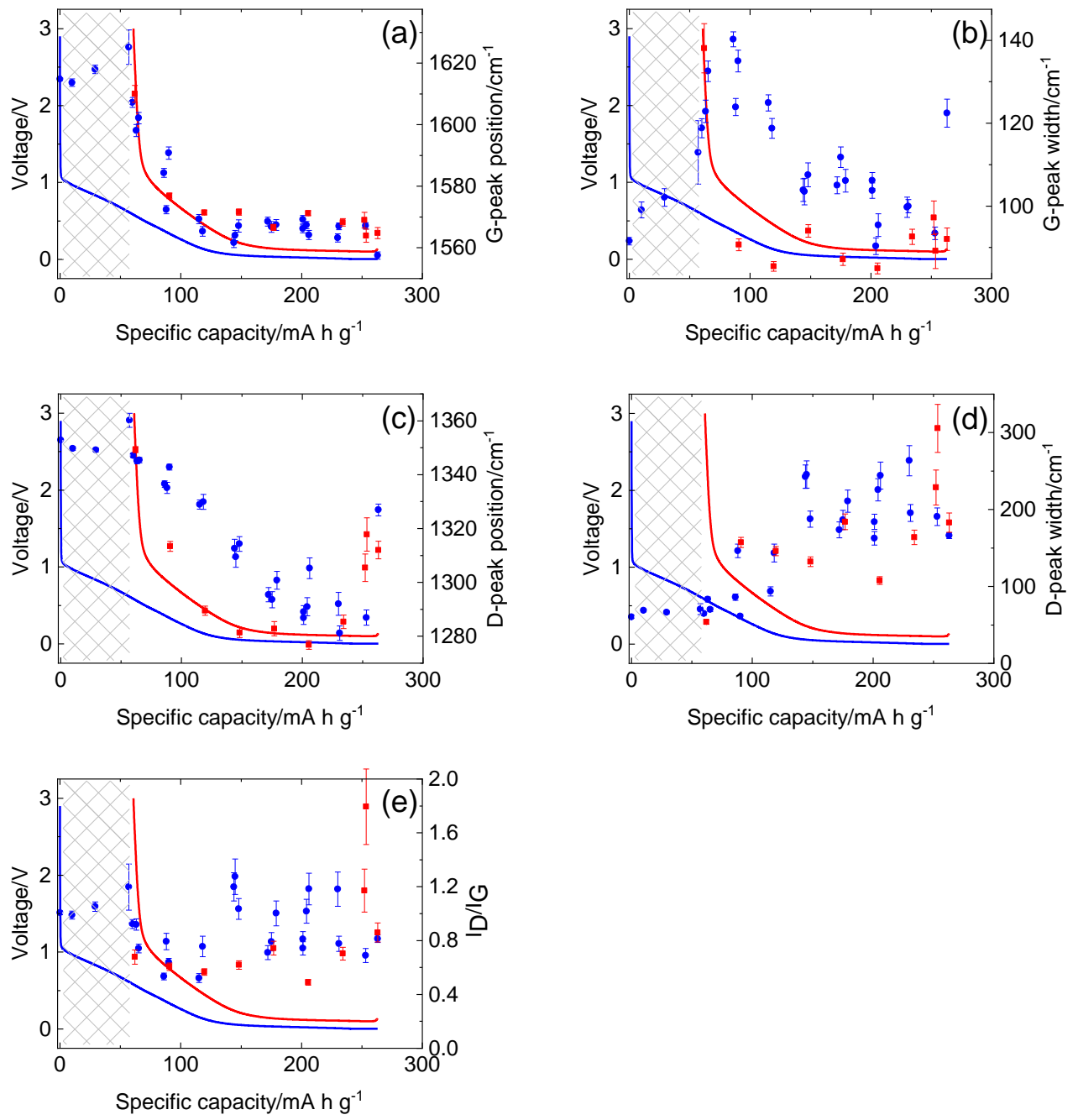

Figure S3 Operando Raman experiment for discharge (sodiation) and subsequent charge (desodiation) of sodium-hard carbon cell on cycle 1; sodiation shown in blue; desodiation shown in red; First Cycle Loss associated with SEI formation shown in gray shading; solid line = voltage profile, discrete points = Raman parameters from fitted G- and D-peaks (blue dots = sodiation, red squares = desodiation): (a) G-peak position, (b) G-peak width, (c) D-peak position, (d) D-peak width and (e) ratio of intensities $\mathrm{I}_{\mathrm{D}} / \mathrm{I}_{\mathrm{G}}$; sodiation was performed by discharging at a constant current of $\mathrm{C} / 5$ to $5 \mathrm{mV}$, followed by a constant voltage hold at $5 \mathrm{mV}$ for 30 minutes; desodiation was performed by a constant current charge at $\mathrm{C} / 5$ to $3 \mathrm{~V} ; \mathrm{C} / 5$ was based on $300 \mathrm{~mA} \mathrm{~h} \mathrm{~g}^{-1}$ for hard carbon. 

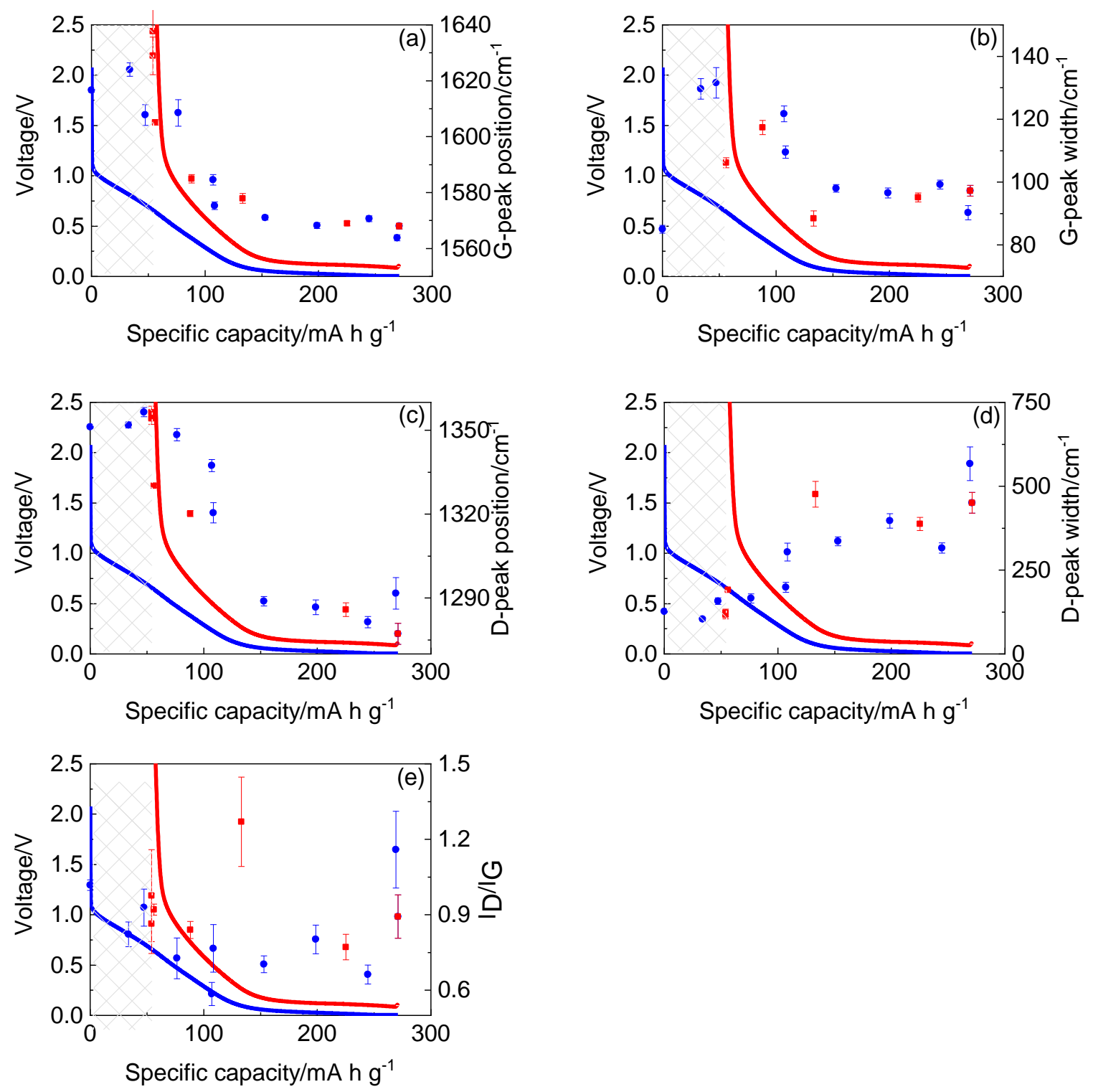

Figure S4 Operando Raman experiment for discharge (sodiation) and subsequent charge (desodiation) of sodium-hard carbon cell on cycle 1; sodiation shown in blue; desodiation shown in red; First Cycle Loss associated with SEI formation shown in gray shading; solid line = voltage profile, discrete points = Raman parameters from fitted G- and D-peaks (blue dots = sodiation, red squares = desodiation): (a) G-peak position, (b) G-peak width, (c) D-peak position, (d) D-peak width and (e) ratio of intensities $\mathrm{I}_{\mathrm{D}} / \mathrm{I}_{\mathrm{G}}$; sodiation was performed by discharging at a constant current of $\mathrm{C} / 3$ to $5 \mathrm{mV}$, followed by a constant voltage hold at $5 \mathrm{mV}$ for 17 minutes; desodiation was performed by a constant current charge at $\mathrm{C} / 3$ to $3.5 \mathrm{~V} ; \mathrm{C} / 3$ was based on $300 \mathrm{~mA} \mathrm{~h}^{-1}$ for hard carbon. 

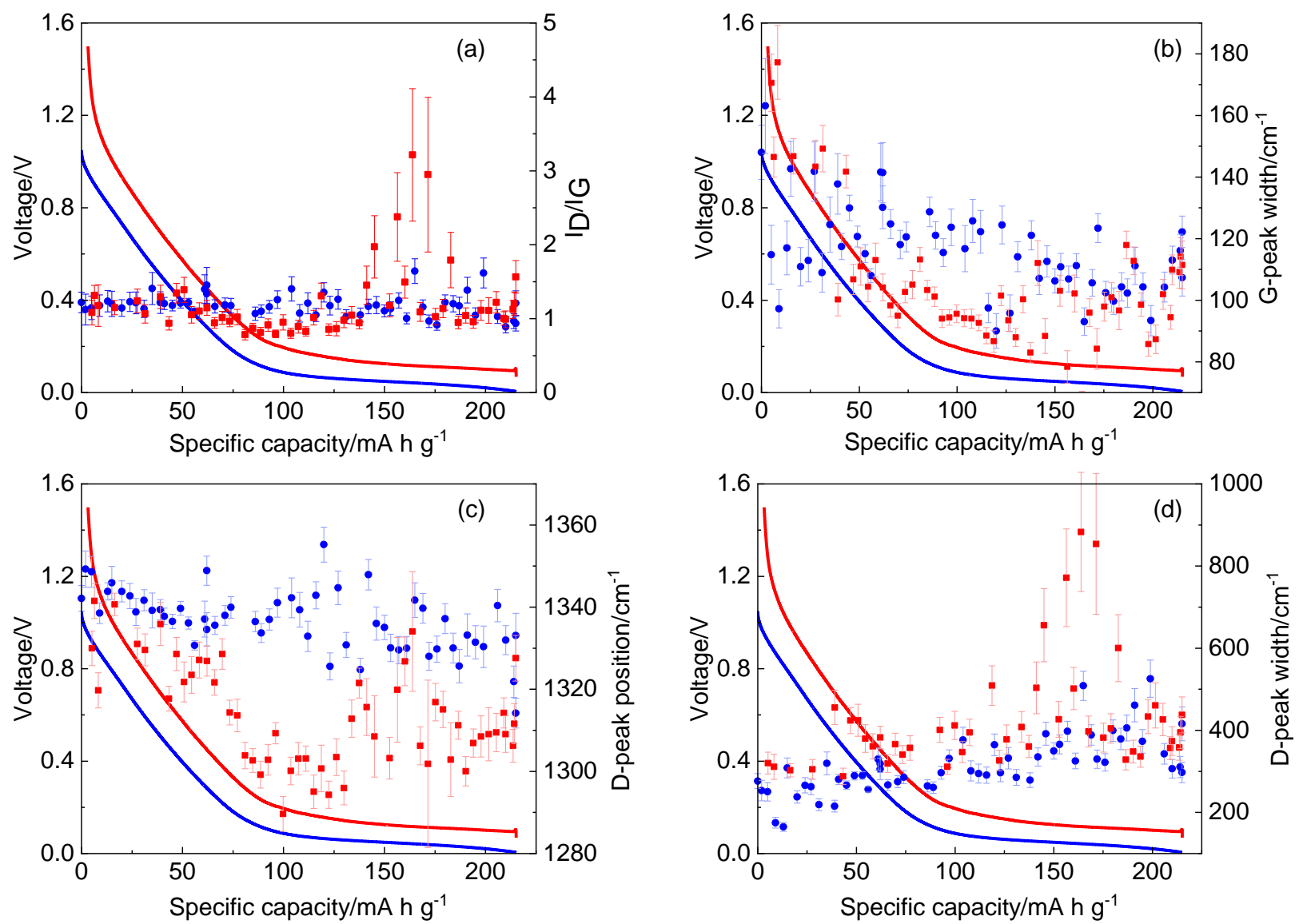

Figure S5 Operando Raman experiment for discharge (sodiation) and subsequent charge (desodiation) on cycle 9 of sodium-hard carbon cell described in Figure 3 and Figure S1; sodiation shown in blue; desodiation shown in red; solid line $=$ voltage profile, discrete points $=$ Raman parameters from fitted G- and D-peaks (blue dots = sodiation, red squares = desodiation): (a) ratio of intensities $\mathrm{I}_{\mathrm{D}} / \mathrm{I}_{\mathrm{G}}$; (b) Gpeak width; (c) D-peak position; (d) D-peak width; sodiation was performed by discharging at a constant current of $\mathrm{C} / 5$ to $5 \mathrm{mV}$ (there was no constant voltage hold) and desodiation was performed by a constant current charge at $\mathrm{C} / 5$ to $1.5 \mathrm{~V}$; $\mathrm{C} / 5$ was based on cycle 1 charge (desodiation) capacity (Figure 3 and Figure S1); G-peak position given in Figure 3. 


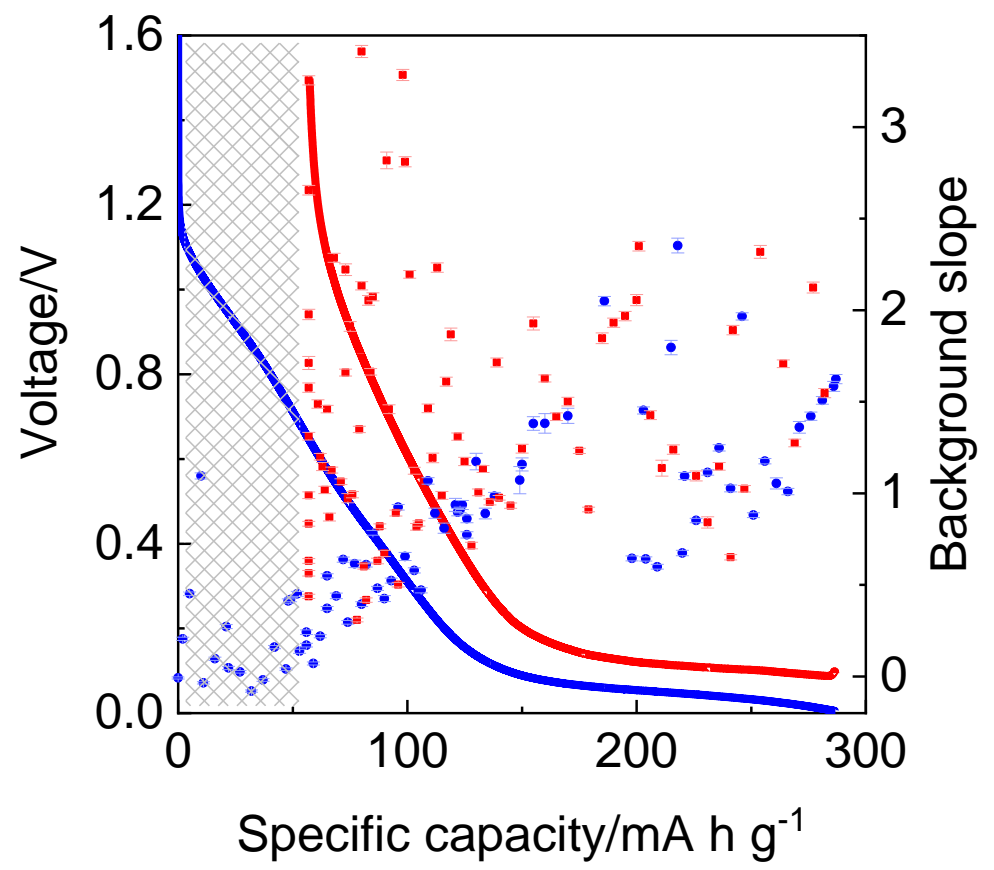

Figure S6 Background slope plotted with voltage profile against specific capacity for Raman spectra recorded during discharge (sodiation) and subsequent charge (desodiation) on cycle 1 of sodium-hard carbon cell described in Figure 3; sodiation shown in blue; desodiation shown in red; solid line = voltage profile, discrete points $=$ background slope (blue dots $=$ sodiation, red squares $=$ desodiation); First Cycle Loss associated with SEI formation shown in gray shading.
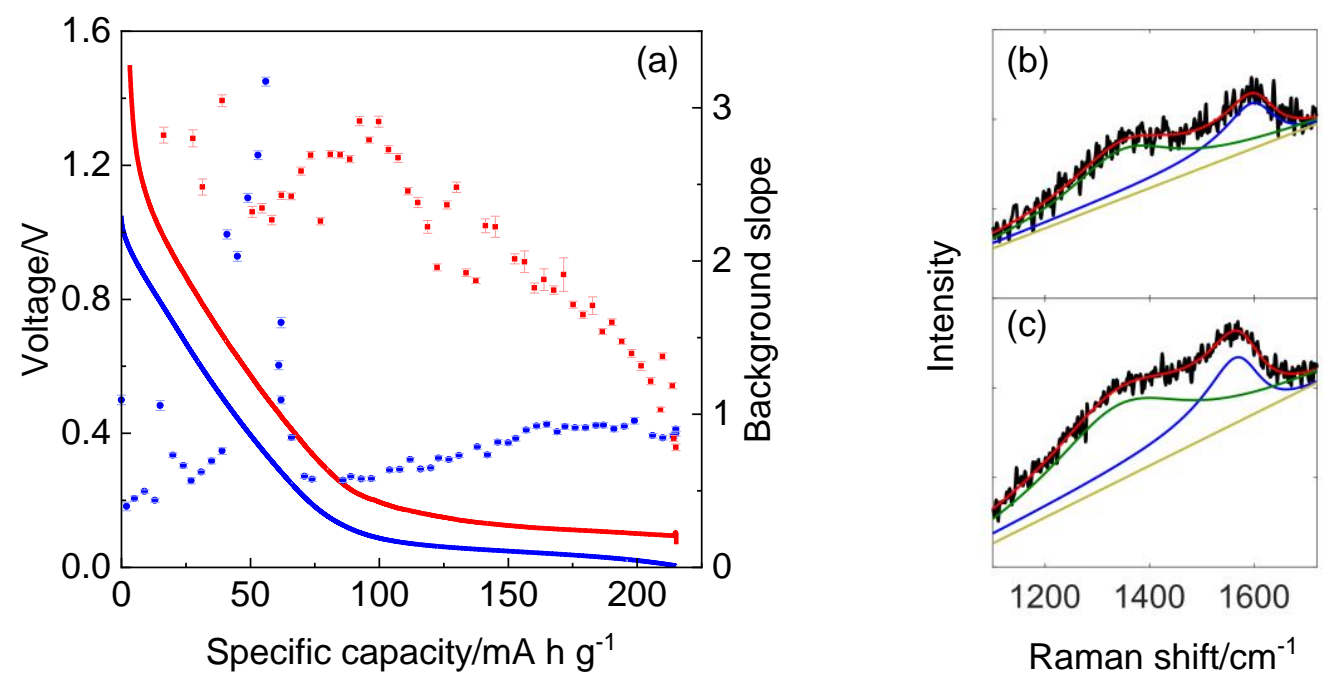
Figure S7 (a) Background slope plotted with voltage profile against specific capacity for Raman spectra recorded during discharge (sodiation) and subsequent charge (desodiation) on cycle 9 of sodium-hard carbon cell described in Figure 3; sodiation shown in blue; desodiation shown in red; solid line $=$ voltage profile, discrete points $=$ background slope $($ blue dots $=$ sodiation, red squares $=$ desodiation); (b, c) selected spectra recorded at different capacities during sodiation on cycle 9: (b) 5 $\mathrm{mA} \mathrm{h} \mathrm{g}{ }^{-1}$, (c) $97 \mathrm{~mA} \mathrm{~h} \mathrm{~g}^{-1}$; data (black), total fit (red), Lorentzian fit (green), Breit-Wigner-Fano fit (blue), background (dark yellow). 